\title{
Qualidade da Atenção em Rede Hierarquizada: A Interface Serviço/Paciente
}

* Docentes do Departamento de Faculdade de Ciências Médicas - Unicamp.
Paulo Roberto de Madureira* Eduardo Mello De Capitani*

Os autores relatam resultados de avaliação de qualidade dos serviços prestados a nivel do ambulatório de adultos do Centro de Saúde-Escola de Paulínia, dentro de um sistema hierarquizado de atençâo à saúde.

Tentando definir parâmetros e procedimentos metodológicos reproduzíveis em outros serviços semelhantes, optam pelo estudo de variáveis relacionadas à assimilação das informações diagnósticas e terapêuticas veiculadas na relação serviço/paciente durante o processo de atendimento, incluindo pré-consultas, consultas de enfermagem $e$ médica, pós-consultas e entrega de medicamentos na farmácia do serviço. Utilizando método de entrevista de pacientes sorteados quinze dias após a consulta, obtivemos dados sobre conceitos diagnósticos, prognósticos, investigação complementar e terapêutica que foram assimilados pelos pacientes. $O$ elevado percentual de pacientes que apreenderam e seguiram as orientaçoes fornecidas $(51 \% ; 72,7 \% ; 87 \% ; 77 \%$, respectivamente) no processo de atendimento, provavelmente, reflete uma certa qualidade técnica do serviço prestado. Concluem pela exeqüibilidade de avaliaçóes periódicas, utilizando esses indicadores, viabilizando ajustes e mudanças mais ágeis nos serviços, visando à melhoria da qualidade.

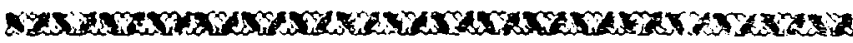

\section{INTRODUÇĀO}

A avaliação da qualidade de serviços ambulatoriais tem trazido na prática problemas metodológicos de importância, tendo em vista características específicas desse tipo de prestação de serviços que a diferenciam do cuidado hospitalar, por exemplo, onde variáveis de utilização são passíveis de controle num processo de investigação (Christoffel, T. e Loewenthal, M., 1977), (Hulka, B. S. e Wheat, J. R., 1985), (Elstein, A. S. e Jason, H., 1970), (Schneeweiss, R. et al., 1983), (Bentsen, B. G., 1976), (MC Guire, C. H. 1985), (Koran, L. M., 1975), (Weiss, G. L., 
1988), (Noren, J. et al., 1980), (Simeant, S., 1983), (Lopes, I. M. et al., 1974), (Deniston, O. L. et al., 1969). Alguns aspectos críticos do cuidado ambulatorial, assim chamados por definirem um umbral de variáveis passíveis de manipulação ou não, podem ser delineados para configurar as dificuldades na abordagem sistemática da prática ambulatorial, quando se visa a sua avaliação (Christofell, T. e Loewenthal, M., 1977). Entre esses aspectos, pode-se citar a nāo-existência de episódio de doença facilmente definida em grande parte da demanda desses serviços (Klotzel, K., 1980), (Smith, G. R. et al., 1986). Conseqüentemente, os pacientes, em geral, não recebem um diagnóstico específico, sendo que os códigos padronizados de doenças existentes rião dão conta desse universo de queixas e problemas por serem baseados num padrão hospitalar de patologias (Schneeweiss, R. et al., 1983), (Koran, L. M., 1975), (Simeant, S., 1983). Outro aspecto crítico diz respeito aos prontuários que não são uniformes, variando conforme o tempo de envolvimento do paciente com o serviço. Outra fonte de variação é proveniente do tipo de médico que atende e registra (Schneeweiss, R, et al., 1983), (Koran, L. M., 1975). A ausência de um controle estrito sobre a aderência às terapêuticas prescritas, por parte do médico ou do serviço, em geral inviabiliza esta variável como parâmetro de qualidade da atenção prestada. A diversidade de propostas de atendimento conforme a política adotada e o tipo de demanda atendida pelos diversos serviços tomam pouco viável, para não dizer impossível, adoção de metodologia básica, universal, para avaliações sistemáticas que possam ser comparáveis (Christoffel, T. e Loewenthal, M., 1977), (Weiss, G. L., 1988), (Eddy, D. M., 1982), (Kronenfeld, J. J., 1978).

Em competente revisão sobre o assunto, Christoffel e Loewenthal, em 1977, discutem detalhadamente alguns sistemas de codificaçōes/classificaçōes dos "problemas doenças" a nível ambulatorial, sugerindo parâmetros para a utilização em prontuários e definições mais precisas dos quadros patogênicos encontrados. Ressaltam que, apesar de antiga, a idéia de se avaliarem serviços ambulatoriais não vingou como sistemática até o momento, tendo-se investido com muito mais audácia na avaliação de qualidade dos serviços hospitalares, em função do impacto desse tipo de atenção na saúde das pessoas (casos graves, intermações por diagnósticos definidos) e da necessidade de correções mais rápidas nas condutas (Christoffel, $T$. e Loewenthal, M., 1977).

Estudando os métodos até hoje utilizados na avaliação de serviços de saúde ambulatoriais, esses autores explicitam três abordagens para o problema. Ao pri- 
meiro método chamam de abordagem estrutural (in put studies) onde se tenta focalizar a quantidade e a qualidade dos recursos alocados para a prestação dos serviços. Esse método tentaria relacionar esses recursos com a "qualidade" do serviço, visando avaliar a adequação dos primeiros. Um segundo grupo de estudos visa estudar o processo de prestação de serviços, tentando aferir como os recursos são utilizados. Estes estudos partiriam do suposto que se as atividades adequadas são realizadas então os resultados serâo os melhores possiveis (Christoffel, T. e Loewenthal, M., 1977). Pressupõem utilização prévia de protocolos de atendimentos e padronizaçōes de condutas (interferência a priori no processo, para garantir resultados). O terceiro método teria como objetivo a gama de resultados conseguidos a partir da atuação dos serviços (out come studies). Utilizam-se de parâmetros e variáveis que mediriam o impacto do serviço a nivel da melhora do estado de saúde dos usuários. Analisam parâmetros tais como mortalidade, morbidade, tempo de permanência no serviço, complicaçôes de terapêuticas; satisfação com o cuidado recebido; incapacidade; estado funcional; mudanças de expectativa de vida; adequação social etc. Nessa linha de estudos de resultados, Willianson, em 1971, propôs a utilização do que chamou de "tópicos de auditoria" (audit topics), tentando restringir e qualificar o número de variaçōes a partir, por exemplo, da avaliação de uma patologia passível de padronização nos seus aspectos diagnósticos e terapêuticos, como a infecção do trato urinário (Willianson, J. W., 1971). O mesmo raciocínio é percorrido por Schroeder, em 1976, quando propõe análise do que chama de "marcadores" (tracers), que seriam o reflexo da atuação do serviço (Schroeder, S. A. e Donaldson, M. S., 1976).

Hulka e Wheat, em 1985, em outra revisão de literatura acerca do tema, tentando responder quais fatores influenciam as pessoas a utilizarem serviços de saúde, dividem os trabalhos até então produzidos em cinco categorias: 1) estudos visando o estado de saúde e a necessidade de utilização de serviços (health status and need);2) características demográficas; 3) disponibilidade de métodos; 4) características de organizaçăo de serviços e 5) mecanismos de financiamento. Utilizando o modelo epidemiológico clássico, os autores ressaltam a questẩo do estudo da "necessidade" como fator de utilizaçāo de serviços de saúde, demonstrando haver uma relação causal entre esse fator e a utilização. Essa relação causal seria dada pela força da relação, podendo-se calcular o risco relativo de se procurar o serviço a partir de um sintoma; pela procedência da relação e pela natureza dose-resposta da relação, sendo que a gravidade das situações atua- 
riam como "dose" e a procura ao serviço como "resposta". Concluem, pela análise de diversos trabalhos nessa linha, que a necessidade de cuidado médico é o maior determinante do uso de serviços de saúde, (Hulka, B. S. e Wheat, J. R., 1985). Outros fatores, estudados dentro das outras quatro categorias de estudo examinadas, seriam de importância secundária. Estreitamente ligado ao estudo da necessidade de utilização está o estudo das repercussões do cuidado médico no estado de saúde. No entanto, a imprecisão dos parâmetros a serem aferidos desnorteia os esforços em definir o que o serviço de saúde trouxe de benefício ao paciente que o procurou.

Outras abordagens, que talvez se enquadrassem no segundo grupo de Christoffel e Loewenthal, tentam partir do processo diagnóstico, como fundamento do ato clínico, para avaliação da qualidade do serviço prestado. Elstein e Jason, em 1970, enfrentaram a questão a partir de situações simuladas que incluiam problemas freqüentes na prática clínica. A artificialidade do método de aferição, no entanto, praticamente impossibilita a definição de parâmetros utilizáveis em outras situações (Elstein, A. S., e Jason, H., 1970).

Por outro lado, em investigaçōes cuja metodologia se utilizava de situações mais próximas de um atendimento real, constataram-se efeitos extremamente curiosos e que vieram se somar ao já imenso rol de variáveis a serem controladas, quando se analisam padrões a partir de processo diagnóstico. Entre outras, Noren et al., em 1980, constataram diferenças na quantidade de informaçōes, no número de exames laboratoriais e de pedidos de Raios- $X$ entre especialistas em clínica médica e médicos generalistas, para o mesmo tipo de problema proposto, sugerindo reflexos na qualidade da atençăo prestada. Ou seja, variáveis quanto à formação profissional devem ser levadas em conta (Koran, L. M., 1975), (Noren, J. et al., 1980). Da mesma forma, resultados dos atendimentos, a partir de diferenças no processo diagnóstico, variarắo conforme a demanda esteja organizada ou não (Christoffel, T. e Loewenthal, M., 1977).

Analisando-se trabalhos que investigaram questões de qualidade de serviços a partir dos registros médicos (Elstein, A. S. e Jason, H., 1970), (Bentsen, B. G., 1976), (Froom, J., 1979), percebe-se que as dificuldades de padronizaçōes metodológicas também são grandes, variando desde o conteúdo dos registros para queixas semelhantes até avaliaçōes distintas dos problemas principais que levaram o paciente ao serviço (Bentsen, B. G. 1976). A questão das definições classificatórias prévias para problemas ambulatoriais reflete-se também nessas análises de registros visando avaliações de qualidade (Christoffel, T. e Loewenthal, 
M., 1977), (Schneeweiss, R. et al., 1983), (Froom, J., 1976).

Numa tentativa de isolar variáveis dependentes de julgamentos de valor como satisfaçāo, mudança na expectativa de vida, adequação social etc e também não trabalhar com parâmetros tipo morbidade, mortalidade, que necessitariam tempo prolongado de observação, características de estabilidade demográfica não existentes no momento, além de controle de praticamente todo o processo de prestação de serviços (padronizaçōes diagnósticas, terapêuticas, classificações nosológicas adequadas etc), optamos, na presente investigação, pela avaliação da qualidade dos serviços na clínica de adultos do Centro de Saúde-Escola de Paulínia (CSEP), a partir de variáveis relacionadas à assimilação das informações diagnósticas e terapêuticas veiculadas na relação paciente/serviço de saúde no processo de atendimento. Nessa relação paciente/serviço de saúde estão contidas as relações médico/paciente e pessoal de enfermagem e farmácia/paciente.

\section{MATERIAL E MÉTODOS}

Paulínia é um município industrial do interior de São Paulo, cuja população estimada para 1987 era de 35.014 habitantes, com $92 \%$ concentrados na zona urbana e $8 \%$ na zona rural. A principal atividade da cidade está ligada à indústria petroquímica, que constitui pólo de atração para os trabalhadores da região.

Desde 1974, através de convênio entre a Secretaria de Saúde do Estado, Prefeitura Municipal e Unicamp, funciona o Centro de Saúde-Escola de Paulínia, com objetivos de prestar assistência médico-sanitária à população e servir de campo de treinamento para alunos e residentes da Faculdade de Ciências Médicas da Unicamp, tentando, desde então, alcançar a tão almejada integração docente-assistencial no âmbito do ensino médico.

Com o aumento da população e conseqüente aumento da demanda de assistência médica, o Centro de Saúde-Escola de Paulínia evoluiu para a criação de três Postos de Saúde em bairros periféricos e a ativação de um hospital com 100 leitos, em meados de 1986, constituindo, dessa forma, um módulo completo, regionalizado e hierarquizado, de prestação de serviços de saúde.

Devido a esse avanço e à implementação da assistência pública no município, a medicina liberal e de convênios médicos não se desenvolveu de forma expressiva, sendo atualmente responsável por pequena parcela de demanda em saúde, não dispondo de todos os níveis de atuação. 
Deste modo, a produção de serviços, a partir deste módulo de saúde, espelha, de maneira bastante realísti$\mathrm{ca}$, as necessidades de saúde desta população. Os dados de cobertura relativos ao ano de 1987 mostram índices de $138,1 \%$ para a faixa de $0-1$ ano; $47,6 \%$, de 1 a 4 anos; $42,9 \%$, na faixa de $5-14$ e $40,9 \%$, na faixa de adultos, com $62,8 \%$ no atendimento obstétrico/pré-natal, para primeiro atendimento. Da mesma forma, a cobertura vacinal para o mesmo ano foi de 96,36\% para poliomielite (Sabin); 97,99\% para vacina tríplice; $107,02 \%$ para sarampo e $78,04 \%$ para $B C G$. $\mathrm{O}$ índice consultas médicas por habitantes, por ano, calculado por faixas etárias, mostrou ser de 3,4 para $0-1$ ano; 3,9 para $1-4$ anos; 1,61 para $5-14$ anos; 2,05 para adultos; 3,15 para ginecologia e 5,99 para obstetrícia/pré-natal. Esses dados, referentes a um ano em que praticamente a rede como um todo estava já consolidada, mostram, de maneira clara, uma cobertura de necessidades bastante adequada, considerando-se que as outras formas de oferta de serviços de saúde têm pouca expressão (Ministério da Previdência e Assistência Social, 1983), (Molain, M. S. A. e Moraes, N. M. A., 1985), (Ministério da Saúde, 1985).

$\mathrm{O}$ atendimento médico realizado no Centro de Saúde-Escola, especificamente, é realizado por graduandos de 6: ano médico e residentes de Medicina Preventiva, com supervisão docente, caso a caso. $O$ serviço se divide em quatro áreas: ambulatório de adultos, pediatria, gineco-obstetrícia e cirurgia, havendo ainda algumas especialidades, como oftalmologia e saúde mental, consideradas estratégicas nesse tipo de serviço. $O$ atendimento é universal e garante o acesso a todos os níveis hierárquicos disponíveis.

Apesar de verificarmos que o Centro de SaúdeEscola de Paulínia e os três postos periféricos funcionam como porta de entrada do sistema como um todo (a nível terciário dispõe-se do Hospital das Clínicas da Unicamp), do ponto de vista quantitativo (ver dados de cobertura), a qualidade de assistência prestada é motivo de preocupação constante da equipe de técnicos envolvida.

Em trabalho anterior (Madureira, P. R. et al., 1989), começamos a analisar alguns aspectos qualitativos da prestação de serviços, na área de adultos do Centro de Saúde-Escola de Paulínia, na tentativa de determinarmos alguns indicadores de qualidade de atenção prestada que servissem para posterior avaliação de rede como um todo.

A manipulação e transformação de dados aparentemente quantitativos de atendimento, como prescrição de medicamentos, pedidos de exames complementares, retomos, encaminhamentos e outros, com índi- 
ces que indicassem a forma de utilização de recursos e revelassem parte do conteúdo dos atendimentos, nos indicaram alguns caminhos, na busca de aferição de qualidade de serviços que fosse adequada ao nosso meio.

O ambulatório de adultos do CSEP, que é alvo de nossa investigação, funciona com uma demanda $70 \%$ agendada e $30 \%$ espontânea, aberta para casos mais urgentes.

A recepção dos pacientes é feita por duas auxiliares de enfermagem, que fazem a triagem para consultas médicas agendadas ou eventuais ou para consultas de enfermagem. As consultas médicas são feitas por internos de 6: ano médico e residentes de Medicina Preventiva. Todos os casos são discutidos, inicialmente, numa sala alocada para esse propósito, para tentar preservar os aspectos transferenciais envolvidos na relação com interno-residente/paciente e, posteriormente, caso haja necessidade, completa-se a discussão no próprio consultório, junto ao paciente, checando-se dados de anamnese e/ou exame físico que ficaram pouco claros na discussão inicial e fazendo-se a orientação final do caso.

A consulta de enfermagem, acima referida como parte do atendimento de adultos, vem sendo realizada desde 1983. Foi criada e desenvolvida no intuito de aliviar as consultas médicas dos retornos repetidos e freqüentes, necessários ao acompanhamento adequado de doentes crônicos, assim como de dar conta de eventuais intercorrências clínicas de outros pacientes já matriculados.

Essas consultas são realizadas por técnicos de enfermagem, enfermeiras universitárias e algumas auxiliares treinadas no próprio serviço, sendo supervisionadas, caso a caso, diretamente pelos docentes da área.

$O$ intercâmbio de informaçōes e posturas frente aos docentes, além de aspectos psicodinâmicos, parece ser o melhor meio de aprendizagem do pessoal auxiliar, permitindo $ı \mathrm{~m}$ reciclar contínuo e regular, além de um contato estreito com os pacientes crônicos que necessitam de um referencial mais constante na equipe de atendimento do que o médico que, no caso do CSEP, a exceção dos docentes, apresenta rotatividade relativamente alta.

No presente trabalho, tenta-se responder à seguinte pergunta: como o contato com serviço de saúde está conseguindo resolver os problemas traduzidos pelos pacientes? Em outros termos, na interface serviço/ paciente, este estará recebendo uma orientação que realmente o encaminhe para a solução de seus problemas? 
Tentando verificar este aspecto, que a nosso ver embute referências à qualidade da atenção prestada, realizamos entrevistas domiciliares com pacientes que procuraram o ambulatório de adultos, cujo roteiro básico encontra-se no Quadro I.

\section{QUADRO I}

Roteiro da Entrevista Domiciliar Individual

NOME:

IDADE: FF:

END:

1. DIAGNÓSTICO - EPIDEMIOLOGIA - PROGNÓSTICO.

Esteve doente?

Onde procurou assistência primeiramente?

Sabe o nome da doença?

$O$ que é?

Como se pega?

É contagiosa?

Como se transmite?

Pode-se prevenir?

Como?

É curável

É controlável?

Qual?

2. EXAMES COMPLEMENTARES.

Foram pedidos exames?

Sabe o nome dos exames?

Como?

Como?

Para que servem?

Como serảo realizados?

Já fez os exames?

Se näo fez, por quê?

3. TERAPÊUTICA

Foram prescritos medicamentos?

Sabe os nomes?

Quantos?

Descrever as funçöes:

Sabe a função?

Sabe a posolcgia?

Tempo de uso:

Tomou? Se não, por quê?

4. SEGUIMENTO/ENCAMINHAMENTO.

Necessita retorno?

Para quando?

Por quê?

Foi encaminhado?

Para onde?

Qual especialidade?

Por quê? 
As entrevistas foram realizadas nos fins de semana, no intuito de atingir-se ambos os sexos igualmente; um entrevistador único foi orientado no sentido de obter dados que respeitassem ao máximo as características culturais de linguagem dos pacientes, anotando, na medida do possivel, expressões literais.

Foram inquiridos pacientes que consultaram o ambulatório de adultos pela primeira vez, evitando possível viés causado por conhecimento prévio de jargōes e procedimentos, gerados por contatos repetidos anteriores com o serviço. $O$ intervalo entre a consulta e a entrevista foi de no mínimo duas e no máximo três semanas, visando avaliar conhecimentos mediados pelo contato com o serviço e que ficaram realmente sedimentados.

O conteúdo da entrevista visava estabelecer o nível de conhecimento que o paciente recebeu/apreendeu em relação à sua doença, gravidade, prognóstico, epidemiologia, se seguiu prescriçōes terapêuticas e/ou realizou procedimentos diagnósticos complementares.

Foram procurados para entrevista 55 pacientes adultos, de ambos os sexos, que procuraram o CSEP para primeira consulta num período de dois meses. O entrevistador não teve qualquer contato prévio com esses pacientes e não sabia de antemão seus diagnósticos, e todos os dados foram contrapostos aos do prontuário.

\section{RESULTADOS E DISCUSSĀO}

$\mathrm{Na}$ Tabela I encontram-se os dados referentes à distribuição dos entrevistados segundo a faixa etária. Nota-se o predomínio relativo de $53,6 \%$ de pacientes dentro da faixa etária de 20 a 50 anos, ou seja, a época produtiva da vida.

Tem-se, dessa forma, uma amostra de pacientes adultos, que foge completamente aos padrōes da demanda de serviços de saúde pública tradicional, em que o predomínio é de mulheres em idade reprodutiva, em busca de atendimento pré-natal. Em nosso grupo de pacientes, houve uma proporção de $38 \%$ de homens e $62 \%$ de mulheres (Tabela II).

Com relação ao funcionamento da rede básica de Paulínia, como porta de entrada do sistema, temos alguns dados indicativos mostrando o CSEP como opção de primeiro atendimento, frente a um problema de saúde, para $62 \%$ dos entrevistados (Tabela III), sendo que $24 \%$ deles procuraram o Pronto Socorro Municipal público, que funciona integrado à rede como serviço de urgência, tendo sido referidos ao CSEP para o atendimento ambulatorial e seguimento. 
TABELA I

Distribuição dos entrevistados, segundo a faixa etária

\begin{tabular}{|c|c|c|c|}
\hline \multicolumn{2}{|c|}{ Faixa Etária } & \multirow{2}{*}{$\frac{\text { N: }}{4}$} & \multirow{2}{*}{$\begin{array}{r}\% \\
7,3\end{array}$} \\
\hline 14 & 20 & & \\
\hline 21 & 30 & 13 & 23,6 \\
\hline 31 & 40 & 12 & 21,8 \\
\hline 41 & 50 & 10 & 18,2 \\
\hline 51 & 60 & 7 & 12,7 \\
\hline 61 & 70 & 5 & 9,2 \\
\hline 71 & 80 & 3 & 5,4 \\
\hline 81 & e + & 1 & 1,8 \\
\hline \multicolumn{2}{|c|}{ TOTAL } & 55 & 100,0 \\
\hline
\end{tabular}

TABELA II

Distribuição dos entrevistados de acordo com o sexo

\begin{tabular}{llc}
\hline Sexo & N: & $\%$ \\
\hline Masculino & 21 & 38,0 \\
Feminino & 34 & 62,0 \\
\hline TOTAL & 55 & 100,0 \\
\hline
\end{tabular}

TABELA III

Distribuição dos pacientes entrevistados, segundo o Serviço de Saúde procurado primeiramente

\begin{tabular}{lrr}
\hline Serviço de Saúde Procurado & N: & $\%$ \\
\hline CSEP & 34 & 62,0 \\
PS/PMP & 13 & 24,0 \\
Outros & 8 & 14,0 \\
\hline TOTAL & 55 & 100,00 \\
\hline
\end{tabular}


A distribuição dos diagnósticos realizados segundo a evolução da patologia, constante da Tabela IV, aponta para uma definiçăo de certo equilíbrio no padrăo nosológico da demanda atendida, havendo praticamente uma equivalência entre patologias agudas e crônicas, indicando que o serviço responde, em certa medida, à estrutura epidemiológica da população-alvo, com abordagem técnica de patologias gerais, aparentemente sem vícios a nível de especialização do atendimento.

A partir da Tabela $V$, vemos os dados referentes aos conceitos pesquisados e assimilados pelo paciente durante 0 ato de atendimento. Foram questionadas noções relativas a diagnóstico, procedimentos complementares, prescriçōes terapêuticas (medicamentos e/ou outras orientaçōes), seguimento (retornos) e encaminhamento.

\section{TABELA IV}

Distribuição dos diagnósticos realizados nos pacientes entrevistados, segundo a evolução da patologia

\begin{tabular}{lrc}
\hline Evoluçäo da Patologia & N: & $\%$ \\
\hline Aguda & 24 & 43,6 \\
Subcrônica & 9 & 16,4 \\
Crônica & 22 & 40,0 \\
\hline TOTAL & 55 & 100,0 \\
\hline
\end{tabular}

\section{TABELA $V$}

Distribuição dos pacientes entrevistados, segundo o conhecimento assimilado quanto à noção diagnóstica

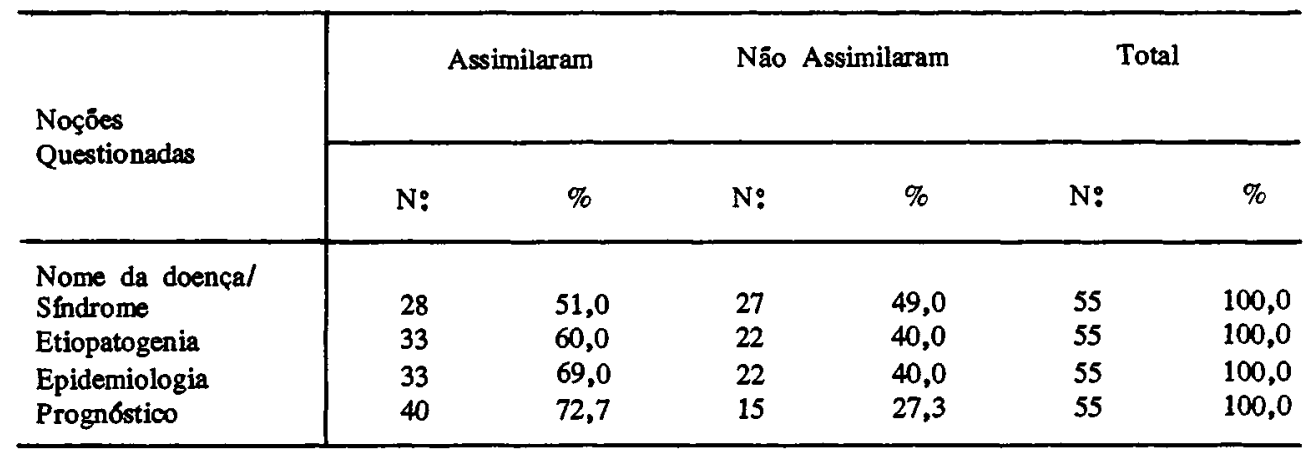


Quanto ao item diagnóstico, foram pesquisadas noções quanto ao "nome de doença", "etiopatogenia", "epidemiologia" e "prognóstico" (Tabela V). Nota-se que os resultados percentuais mostram-se progressivamente favoráveis conforme se caminha do conhecimento do "nome da doença" para "prognóstico". Entendemos que, em termos de importância para a relação médico/paciente e/ou serviço/paciente, quanto a confiança, adesão às consultas e presença a retomos, o prognóstico pode ser considerado, também, como a dimensão mais importante buscada por eles dentro do "diagnóstico" que lhes é dado. Nesse sentido, os resultados apontam na direção correta. Quanto mais o paciente souber sobre o seu prognóstico, independente do nome que se dê a seu diagnóstico, mais "qualificado" ele se toma como paciente, funcionalmente falando. Noções de etiopatogenia e epidemiologia fazem parte essencial desse processo de compreensão global do seu padecimento a partir do prognóstico.

Nas Tabelas VI e VII, apresentamos os dados referentes ao entendimento de informaçōes quanto à realização de procedimentos de auxílio diagnóstico. $\mathrm{Na}$ Tabela VI, nota-se, inicialmente, que apenas $58 \%$ dos pacientes necessitaram de algum exame complementar (32 pacientes). Destes 32, $79 \%$ realizaram esses exames, sendo que $87 \%$ haviam assimilado as informaçōes/orientaçōes referentes aos exames.

Da mesma forma, temos na Tabela VIII dados referentes à prescrição de medicamentos. Dos 55 entrevistados, $35(63 \%)$ tiveram necessidade de algum tipo de prescrição medicamentosa. Destes 35, 28 (80\%) tinham boas noções sobre função e posologia das drogas, sendo que $27(77 \%)$ as tinham usado adequadamente.

\section{TABELA VI}

Distribuição clos pacientes consultados, segundo o uso de procedimentos de auxílio diagnóstico

Exames Solitados

\begin{tabular}{l|cc}
\hline \multirow{2}{*}{ Sim } & $\mathrm{N}:$ & $\%$ \\
\cline { 2 - 3 } Não & 32 & 58,0 \\
\hline TOTAL & 23 & 42,0 \\
\hline
\end{tabular}




\section{TABELA VII}

Distribuição dos pacientes entrevistados, segundo o conhecimento assimilado quanto aos procedimentos de auxílios diagnósticos solicitados

Realizaram os exames solicitados

Informaçōes orientaçōes sobre os exames

\begin{tabular}{crrrr} 
& N: & $\%$ & N: & $\%$ \\
\hline Sim & 23 & 79,0 & 28 & 87,0 \\
Näo & 9 & 21,0 & 4 & 13,0 \\
\hline TOTAL & 32 & 100,0 & 32 & 100,0 \\
\hline
\end{tabular}

\section{TABELA VIII}

Distribuição dos pacientes entrevistados, segundo prescrição de terapêutica medicamentosa

\begin{tabular}{l|cc}
\hline & \multicolumn{2}{|c}{ Prescrição de Medicamentos } \\
& N: & $\%$ \\
\hline Sim & 35 & 63,0 \\
Não & 20 & 37,0 \\
\hline TOTAL & 55 & 100,0 \\
\hline
\end{tabular}

*Dados de entrevistas coincidentes com dados do prontuário.

Chama a atenção o percentual relativamente baixo de pacientes com prescrição de medicamentos (63\%), - que pode caracterizar a existência de uma boa relação médico/paciente ou serviço/paciente, em que outros tipos de prescriçōes (dietéticas e fisioterápicas, por exemplo) têm peso importante, podendo refletir índice 
de qualidade de atendimento. Nesse mesmo sentido, tendo os altos índices de entendimento quanto às noçōes de função e posologia, além do uso realmente adequado do que foi prescrito $(80 \%$ e $77 \%$ ), que reforçam essa idéia de boa relação serviço/paciente (que inclui a passagem do paciente pela pré e pós-consulta e explicaçōes adicionais ao nível da farmácia do CSEP na entrega do produto).

\section{TABELA IX}

Distribuiçāo dos pacientes entrevistados, segundo conhecimento assimilado quanto à prescrição recebida

Noçōes sobre fun-

ção e Posologia

Uso Adequado

\begin{tabular}{lcccr}
\hline & N: & $\%$ & N: & $\%$ \\
\hline Sim & 28 & 80,0 & 27 & 77,0 \\
Năo & 7 & 20,0 & 8 & 23,0 \\
\hline TOTAL & 35 & 100,0 & 35 & 100,0 \\
\hline
\end{tabular}

$\mathrm{Na}$ Tabela X, verificamos o percentual de necessidade de retornos dos pacientes (78\%) e de encaminhamentos às especialidades $(11 \%)$. $\mathrm{O}$ al to índice de retornos deve-se à estrutura de atendimento que privilegia a freqüência do paciente ao serviço em detrimento de medicamentos sintomáticos em exagero ou pedidos desnecessários de exames complementares. Esse percentual de encaminhamentos $(11 \%)$ nos parece próximo do real, tendo em vista a complexidade relativa das patologias que ocorreram ao serviço e ao funcionamento adequado das referências a nível secundário e terciário. 


\section{TABELA $X$}

Distribuição dos pacientes entrevistados, segundo o seguimento e/ou encaminhamento a partir da consulta

\begin{tabular}{|c|c|c|c|c|}
\hline & \multicolumn{2}{|c|}{$\begin{array}{l}\text { Necessitaram } \\
\text { Retorno }\end{array}$} & \multicolumn{2}{|c|}{$\begin{array}{l}\text { Encaminhamento a } \\
\text { Especialidades }\end{array}$} \\
\hline & N: & $\%$ & $\mathrm{~N}:$ & $\%$ \\
\hline Sim & 43 & 78,0 & 6 & 11,0 \\
\hline Não & 12 & 22,0 & 49 & 89,0 \\
\hline TOTAL & 55 & 100,0 & 55 & 100,0 \\
\hline
\end{tabular}

\section{CONCLUSÕES}

Tendo em vista a carência de recursos na área de prestação de serviços de saúde no país, refletida nas diversas formas organizadas para este fim a nível público, implicando diversidade de recursos humanos, tecnologia apropriada, objetivos finais, clientela (padrāo de demanda) etc, cremos que qualquer tentativa de aferição de padrōes de qualidade da atenção prestada a nível primário não poderá depender, no atual estágio organizativo do setor, de controle de variáveis epidemiológicas, que se mostram prematura e objetivamente "incontroláveis", como nível de formação de equipe técnica, estabilidade trabalhista da equipe, organização de demanda, aderência da clientela às condutas terapêuticas lato sensu "impacto" das ações a nível de morbidade a curto prazo etc.

Por outro lado, acreditamos que aspectos tradicionalmente contidos na relação médico/paciente e/ou serviço/paciente, incluindo toda a equipe de atendimento, que constituem parte das atividades-meio no processo de prestação de serviços, possam ser aferidos, de maneira controlada e sistemática, através de amostragem da demanda atendida, visando justamente destacar os aspectos qualitativos dessa atenção, que mantém certa independência das variáveis citadas anterior- 
mente. A utilização de patologias ou "problemas" específicos, passíveis de maior padronização da equipe nos aspectos diagnósticos e de conduta, tenderia a melhor controlar essas mesmas variáveis. A sistematização deste tipo de avaliação amostral, com regularidade mantida, serviria, ademais, como feedback constante para reformulação de aspectos de organização da equipe e de incorporação de tecnologia e recursos humanos ao serviço.

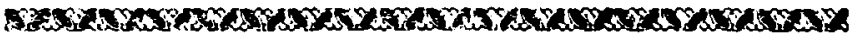

The authors report results from investigation of quality in an adult ambulatory service in Paulinia, SP, Brazil, in a planned health sistem.

Trying to define indicators or monitors able to be reproduced methodologicaly in other services, they choose some variables related to the assimilation of diagnostic, prognostic, subsidiary examination and terapeutic informations by the patient during the consult process into the service. Through an interview of patients a fortnight from the consult day, they were asked about the understanding of concepts that should be passed to them in the service. The high percentage of patients that followed and understood the informations given $(51 \% ; 72,7 \%$; $87 \% ; 77 \%)$ probably reflects the tecnical quality of the service. They conclude that, nowadays, this kind of avaliation is feasible in primary and secondary ambulatory services in Brazil, allowing quick administrative changes trying to improve quality.

\section{REFERÊNCIAS BIBLIOGRÁFICAS}

BENTSEN, B. G. - The accuracy of recording patient problems in family practice. J. Med. Education, 51: 311-316, 1976.

CHRISTOFFEL, T. e LOEWENTHAL, M. - Evaluating the quality of health care: a review of emerging methods. Med. Care, 15 (11): 877-897, 1977.

DENISTON, O. L. et al. - Evaluación del rendimento de programas de salud. Bol. ofic. Sanit. Panan. 67: 389-399, nov. 1969.

EDDY, D. M. - Clinical policies and the quality of clinical practice. N. Eng. J. Med. 307 (6); 343-347, 1982.

ELSTEIN, A. S. e JASON, H. - Estudio del proceso de diagnóstico médico: método y resultados preliminares. Educación Med. y Salud, 4: 355, 1970.

Cadernos de Saúde Pública, RJ, 6(2): 158-174, abr/jun, 1990 
FROOM, J. The international classification of health problems for primary care. Med. Care, 14 (5): 450-454, 1976.

HULKA, B. S. e WHEAT, J. R. - Patterns of utilization: the patient perspective. Med. Care, 23 (5): 438-460, 1985.

KLOTZEL, K. - Clinica Médica: raciocinio e conduta. Editora Pedagógica Universitária. EPU. São Paulo, 1980.

KORAN, L. M. - The reliability of clinical methods, data and judgments, 1st part. N. Engl. J. Med., 293 (13): 642-646, 1975.

KORAN, L. M. - The reliability of clinical methods, data and judgments, 1st part. N. ENGL. J. Med., 293 (14): 695-701, 1975.

KRONENFELD, J. J. - Provider variables and the utilization of ambulatory care services. J. Health Soc. Behavior, 19:68-76, 1978.

LOPES, I. M. et al. - Morbilidad ambulatoria en el aduito: servicio salud metropolitano norte. Cuadernos Med. Sociales, 25: 23-29, 1974.

MADUREIRA, P. R, et al. - Avaliação da Qualidade de Atenção à Saúde na Rede Básica. Cadernos de Saúde Pública, 5 (1): 45-59, 1989.

MC GUIRE, C. H, - Medical Problem - Solving: a critique of the literature. J. Med. Education, 60: 587-595, 1985.

MINIST. PREV. ASSIST. SOCIAL - Reorientação da assistência à saúde no âmbito da previdência social. Documento Oficial, 1983.

MINIST. DA SAÚDE - Instrumento de avaliaçăo para centros e postos de saúde. Centro de Docum. ed. Minist. Saúde Brasilia-DF., 1985.

MOLAIN, M. S. A., e MORAES, N. M. A. - Orientação para organização de Centros de Saúde: o planejamento local. Centro de Docum. ed Minist. Saúde - Brasilia-DF., 1985.

NOREN, J. et al. - Ambulatory medical care: a comparison of internists and family - general practitioners. N. Engl. J. Med., 302 (1): 11-16, 1980.

SCHNEEWEISS, R. et al. - Diagnosis clusters: a new tool for analysing the content of ambulatory medical care. Med. Care, 21 (1): 105-116, 1983.

SCHROEDER, S. A. e DONALDSON, M. S. - The feasibility of and outcome approach to quality assurance: a report from one H. M. O., Med. Care, 14: 49, 1976.

SMITH, G. R, et al. - patients with multiple unexplained symptoms. Arch. Int. Med., 146: 69-72, 1986.

SIMEANT, S. - Estudio de la capacidad resolutiva de la demanda en atención de morbilidad a nivel primario. Cuadernos Med. Sociales. 24: 170-185, 1983.

WEISS, G. L. - Patient satisfaction with primary medical care. Med. Care. 26 (4): 393-392, 1988.

WILLIANSON, J. W. - Evaluating the quality of patient care: a strategy relating outcome and process assesment. Jama, 218: 564,1971 . 